\title{
Hormetic Effects of Mixtures of Dimethachlone and Prochloraz on Sclerotinia sclerotiorum
}

\author{
Ran Zhang, Yuchao Zhang, Qianru Xu, Jianhong Li, and Fuxing Zhu ${ }^{\dagger}$ \\ College of Plant Science and Technology, Huazhong Agricultural University, Wuhan, 430070, China
}

\begin{abstract}
Previous studies showed that dimethachlone has significant hormetic effects on phytopathogenic fungus Sclerotinia sclerotiorum. The present study investigated hormetic effects of mixtures of dimethachlone and prochloraz on mycelial growth and virulence of two dimethachloneresistant isolates of $S$. sclerotiorum. The stimulatory dimethachlone dosage range was around 1 to $100 \mu \mathrm{g} / \mathrm{ml}$ in potato dextrose agar (PDA) medium for mycelial growth of the two isolates, and dimethachlone at 10 and $50 \mu \mathrm{g} / \mathrm{ml}$ had the maximum percent stimulations of 80.6 and $19.3 \%$ for isolates JMS14 and HLJ4, respectively. Prochloraz at 0.0003 and $0.002 \mu \mathrm{g} / \mathrm{ml}$ had the maximum percent stimulations of 9.3 and $11.1 \%$ for isolates JMS14 and HLJ4, respectively. However, dimethachlone and prochloraz mixed at their respective stimulatory concentrations had the maximum percent stimulations of 48.1 and 9.3\% for isolates JMS14 and HLJ4, respectively. After the mycelia with increased and inhibited growth on fungicide-amended PDA were subcultured on PDA without fungicide, mycelial growth for the second generation increased compared with the nontreated control. After the mycelia grown on fungicide-amended PDA were inoculated on rapeseed leaves, the amplitude of virulence stimulation was much greater than that of mycelial growth on PDA amended with fungicide, and the inhibited mycelia also showed substantially increased virulence on leaves. The mixture of dimethachlone at $100 \mu \mathrm{g} / \mathrm{ml}$ and prochloraz

at $0.03 \mu \mathrm{g} / \mathrm{ml}$ in PDA inhibited mycelial growth of isolate JMS14 by $59.4 \%$; however, after the inhibited mycelia were inoculated on rapeseed leaves, virulence was stimulated by $69.0 \%$. Spraying sublethal doses of dimethachlone and prochloraz on rapeseed leaves also exhibited significant stimulatory effects on virulence. For isolate JMS14, the stimulatory concentration ranges for dimethachlone and prochloraz were around 1 to $600 \mu \mathrm{g} / \mathrm{ml}$ and 0.0003 to $0.18 \mu \mathrm{g} / \mathrm{ml}$, respectively. The fitted curve of virulence stimulation for the mixture of dimethachlone and prochloraz shifted to the left on the $x$ axis, denoting dose-additive interactions between the two fungicides with regard to virulence stimulation. Spraying dimethachlone alone at 10 to $50 \mu \mathrm{g} /$ $\mathrm{ml}$ had significant stimulations on virulence, whereas prochloraz alone at 10 to $50 \mu \mathrm{g} / \mathrm{ml}$ had significant inhibitory effects on virulence, and the mixture of dimethachlone and prochloraz at the concentration ratio of 1:1 had greater inhibitory effects than prochloraz alone, indicating dose-additive interactions for the inhibitory effects. Dimethachlone and prochloraz and their mixtures increased tolerance of mycelia to hydrogen peroxide. Dimethachlone significantly increased, whereas prochloraz reduced mycelial cell membrane permeability, and the mixture of the two fungicides had effect-additive interactions with respect to effects on cell membrane permeability. These studies will advance our understanding of hormesis of fungicide mixtures.
\end{abstract}

Sclerotinia sclerotiorum is an agriculturally important fungal pathogen and can infect more than 400 plant species, including many economically important crops such as oilseed rape (Brassica napus), soybean (Glycine max), sunflower (Helianthus annuus), and many vegetables (Boland and Hall 1994; Bolton et al. 2006). S. sclerotiorum often causes serious losses in yield and quality of many crops around the world. Owing to limited genetic resources for resistance to this pathogen, fungicides remain the principal tool for management of $S$. sclerotiorum (Derbyshire and Denton-Giles 2016).

Among the fungicides used to control $S$. sclerotiorum, the benzimidazole fungicide carbendazim is one of the earliest registered fungicides and still is the most widely used fungicide in China. Extensive applications of carbendazim inevitably lead to resistance development. High levels of carbendazim resistance in S. sclerotiorum began to be reported in eastern China in the later 1990s and early 2000 s, and in some cases, control failures of carbendazim were recorded (Pan 1998; Shi et al. 2000; Zhang et al. 2003). Therefore, the dicarboximide fungicide dimethachlone was recommended in the early 2000s to control S. sclerotiorum in eastern China. Several years after the introduction of dimethachlone for control of this pathogen, reduced sensitivity was first reported in 2009 in isolates

${ }^{\dagger}$ Corresponding author: Fuxing Zhu; E-mail: zhufuxing@mail.hzau.edu.cn

Funding: This study was supported by the National Natural Science Foundation of China (31371964)

Accepted for publication 27 September 2018.

() 2019 The American Phytopathological Society collected from Jiangsu Province of eastern China (Ma et al. 2009). Five years later, different levels of dimethachlone resistance were reported in northwestern, northeastern, and central China (Zhou et al. $2014 \mathrm{~b}, \mathrm{c})$. Mixtures and rotations of fungicides with different modes of action are effective application tactics to delay and manage fungicide resistance (Brent and Hollomon 2007). The demethylation inhibitor (DMI) prochloraz is a highly effective systemic fungicide with broad-spectrum activity (Vinggaard et al. 2006). The toxicity and control efficacy of prochloraz against $S$. sclerotiorum have been reported (Ren et al. 2010; Sun et al. 2015; Zhang et al. 2018). The mixture of dimethachlone and prochloraz may provide an ideal and effective fungicide combination for control of $S$. sclerotiorum.

In the process of monitoring dimethachlone resistance in field isolates of $S$. sclerotiorum, we found that dimethachlone at sublethal doses has statistically significant stimulatory effects on growth and virulence of $S$. sclerotiorum. The stimulation amplitude for dimethachlone-resistant isolates is much greater than that for sensitive ones, and the dose range with stimulatory effects for dimethachlone-resistant isolates is higher than that for sensitive ones (Zhou et al. 2014a). Our subsequent studies also showed that the benzimidazole fungicide carbendazim (Di et al. 2015, 2016b), the quinone outside inhibitor fungicide trifloxystrobin (Di et al. 2016a), and the DMI fungicide flusilazole (Lu et al. 2018a, b) have significant stimulatory effects on the virulence of S. sclerotiorum, and carbendazim also has stimulatory effects on the virulence of $\mathrm{Bo}$ trytis cinerea (Cong et al. 2018). Beside our studies, stimulatory effects of fungicides have been reported on many species of plant pathogens, including the DMI fungicide prothioconazole on the production of the mycotoxin deoxynivalenol by Fusarium graminearum (Audenaert et al. 2010), the benzimidazole fungicide thiabendazole on spore germination of Penicillium expansum (Baraldi et al. 2003), the carbamate fungicide propamocarb and the quinone inside 
inhibitor fungicide cyazofamid on mycelial growth of Pythium aphanidermatum (Flores and Garzón 2013), the phenylamide fungicide mefenoxam on mycelial growth and virulence of $P$. aphanidermatum (Garzón et al. 2011), and the phenylamide fungicide metalaxyl on mycelial growth of Phytophthora infestans (Zhang et al. 1997). These stimulatory activities of fungicides on various plant pathogenic fungi and oomycetes belong to a biological concept widely known as hormesis, which describes the biphasic dose-response relationships characterized by high-dose inhibition and low-dose stimulation (Calabrese 2015a). Hormetic responses have been reported across a broad range of biological models and a great diversity of agents (Calabrese 2013, 2015a, b, 2016a, b; Calabrese and Blain 2011).

Fungicide hormesis has profound implications for fungicide applications and management of plant pathogens. Under field conditions, fungicides applied at recommended dosages may become sublethal to some strains of pathogens owing to the drift of droplets, degradation of the active ingredients of fungicides, and different penetration through crop canopies (Garzón and Flores 2013). For the fungicideresistant pathogens, the recommended dosages are actually sublethal and may stimulate rather than inhibit their growth and virulence. Characterization of the hormetic effects of fungicides is essential for designing judicious strategies for fungicide applications. The DMI fungicide prochloraz is highly efficacious against $S$. sclerotiorum (Zhang et al. 2018), and with the occurrence of dimethachlone resistance (Zhou et al. 2014b), mixtures of dimethachlone and prochloraz may be employed to control this pathogen and to delay the development of dimethachlone resistance. Although there have been some studies on hormetic effects of fungicides on S. sclerotiorum (Di et al. 2015; Lu et al. 2018a, b; Zhou et al. 2014a), quantification of hormetic effects of fungicide mixtures on $S$. sclerotiorum is needed. The objectives of the present study were to (i) determine hormetic effects of mixtures of dimethachlone and prochloraz on mycelial growth and virulence of $S$. sclerotiorum, and (ii) assess the interactions between dimethachlone and prochloraz with respect to virulence hormesis.

\section{Materials and Methods}

Isolates of S. sclerotiorum. Two isolates of S. sclerotiorum used in the present study were collected from Heilongjiang Province of Northeast China. The mature sclerotia of isolates HLJ4 and JMS14 were sampled from diseased soybean (G. max) plants in 2011 and 2014, respectively. Both isolates were found to be resistant to the dicarboximide fungicide dimethachlone, and the concentrations that reduce mycelial growth by $50 \%$ ( $\mathrm{EC}_{50}$ values) of dimethachlone for the two isolates HLJ4 and JMS14 were 327.8 and $194.2 \mu \mathrm{g} / \mathrm{ml}$, respectively, and $\mathrm{EC}_{50}$ values of prochloraz for the two isolates were 0.063 and $0.066 \mu \mathrm{g} / \mathrm{ml}$, respectively.

Fungicides. Technical-grade prochloraz (97.0\% active ingredient [a.i.], Hubei Kang Bao Tai Fine-Chemical Co., China) was dissolved in $99.5 \%$ methanol to produce a stock solution of $500 \mu \mathrm{g} / \mathrm{ml}$. Technical-grade dimethachlone (96.2\% a.i., Wenzhou Pesticide Co., China) was dissolved in methanol to generate a stock solution of $10,000 \mu \mathrm{g} / \mathrm{ml}$. These stock solutions were stored at $4^{\circ} \mathrm{C}$ for no more than 2 weeks before being serially diluted for subsequent experiments.

Stimulatory effects of dimethachlone and prochloraz on mycelial growth of $S$. sclerotiorum on potato dextrose agar (PDA). The method was adopted from Cong et al. (2018) and Zhou et al. (2014a). Stock solutions of dimethachlone at 10,000 and $40,000 \mu \mathrm{g} / \mathrm{ml}$ and of prochloraz at $1,000 \mu \mathrm{g} / \mathrm{ml}$ were prepared in methanol. The stock solutions of dimethachlone were further diluted with methanol to 100 , 200, 500, 1,000, 2,000, 5,000, 10,000, and 20,000 $\mu \mathrm{g} / \mathrm{ml}$ for isolate HLJ4 and to 10, 30, 100, 300, 1,000, 3,000, 10,000, and 30,000 $\mu \mathrm{g} / \mathrm{ml}$ for isolate JMS14. The stock solution of prochloraz was diluted with methanol to $0.02,0.04,0.1,0.2,0.4,1,2,4$, and $8 \mu \mathrm{g} / \mathrm{ml}$ for isolate HLJ4 and to $0.003,0.01,0.03,0.1,0.3,1,3$, and $9 \mu \mathrm{g} / \mathrm{ml}$ for isolate JMS14. For fungicide mixtures, dimethachlone and prochloraz were mixed at ratios approximately according to their $\mathrm{EC}_{50}$ values. The mixtures of dimethachlone and prochloraz for isolate HLJ4 were prepared by diluting the stock solution of each fungicide to concurrently contain the two fungicides at the following concentrations: 100 (dimethachlone) +0.02 (prochloraz), $200+0.04,500+0.1,1,000+$ $0.2,2,000+0.4,5,000+1,10,000+2,20,000+4$, and 40,000+ $8 \mu \mathrm{g} / \mathrm{ml}$. For isolate JMS14, the fungicide mixtures concurrently contained the two fungicides at the following concentrations: 10 (dimethachlone) +0.003 (prochloraz), $30+0.01,100+0.03,300+$ $0.1,1,000+0.3,3,000+1,10,000+3$, and $30,000+9 \mu \mathrm{g} / \mathrm{ml}$. These dilutions of each fungicide and their mixtures were added to autoclaved PDA at about $55^{\circ} \mathrm{C}$ in a volume ratio of 1:99. The PDA medium amended with methanol alone was used as the nontreated control. Inverted mycelial plugs ( $5 \mathrm{~mm}$ in diameter) cut from fresh margins of 2-day-old colonies were transferred onto PDA plates prepared as described above. After the PDA plates had been incubated at $23^{\circ} \mathrm{C}$ for $48 \mathrm{~h}$ in the dark in a growth chamber, mycelial growth was measured. Percent stimulations were calculated by the following formula: percent stimulation $(\%)=[($ colony diameter of the treated colony diameter of the control)/colony diameter of the control] $\times$ $100 \%$. The experiment was conducted in triplicate (three PDA plates for each concentration-isolate combination) and repeated independently eight times.

Stimulatory effects of the first-generation growth on fungicideamended PDA on the second-generation mycelial growth on PDA. Fungicide-amended PDA was prepared as described above. After mycelia of the two isolates had been grown on fungicideamended PDA for $48 \mathrm{~h}$ at $23^{\circ} \mathrm{C}$, mycelial plugs were cut with a 5 -mm-diameter cork borer from the actively growing margins of colonies. Mycelial plugs cut from fungal culture colonies grown on PDA amended with the same volume of methanol as that of fungicide dilutions were used as nontreated controls. The fresh mycelial plugs were inoculated on PDA medium without any fungicide or organic solvent. After the PDA plates had been incubated for $48 \mathrm{~h}$ in the dark at $23^{\circ} \mathrm{C}$, the diameter of each mycelial colony was measured twice at right angles. Percent stimulations were calculated with the formula provided in the previous section. The experiment was conducted in triplicate and repeated independently five times.

Stimulatory effects of mycelial growth on fungicide-amended PDA on the virulence of $S$. sclerotiorum to detached leaves of rapeseed plants. The method for this experiment was adopted from Lu et al. (2018b). Fungicide-amended PDA and mycelial plugs were prepared as described above. Leaves of about 9 to $12 \mathrm{~cm}$ in diameter were detached from 4-week-old rapeseed plants growing in the greenhouse at $25^{\circ} \mathrm{C}$. The detached leaves were rinsed with tap water, air dried for $1 \mathrm{~h}$, and transferred to $15-\mathrm{cm}$-diameter Petri dishes lined with wetted filter paper to maintain high humidity. Leaves were inoculated on the adaxial surface with mycelial plugs cut from actively growing margins of mycelial colonies growing on fungicideamended PDA. The inoculated leaves were incubated at $25^{\circ} \mathrm{C}$ for $48 \mathrm{~h}$ in a growth chamber with relative humidity maintained at approximately $85 \%$. The diameter of each lesion was measured twice at right angles. The experiment was conducted in triplicate (three leaves for each treatment) and repeated independently three times.

Stimulatory effects of spraying dimethachlone and prochloraz mixtures on the virulence of $S$. sclerotiorum to rapeseed leaves. The method for this experiment was adopted from Cong et al. (2018) and Di et al. (2016a). The stock solutions of dimethachlone and prochloraz were first diluted with methanol to a series of concentrations and then diluted 100 -fold with $0.1 \%$ Triton X-100 in water. For isolate HLJ4, the final concentrations of dimethachlone were $2,10,50,200$, and $1,000 \mu \mathrm{g} / \mathrm{ml}$, and the final concentrations of prochloraz were $0.0004,0.002,0.01,0.04$, and $0.2 \mu \mathrm{g} / \mathrm{ml}$. Dimethachlone and prochloraz were combined at the following concentrations: 2 (dimethachlone) +0.0004 (prochloraz), $10+0.002,50+$ $0.01,200+0.04$, and $1,000+0.2 \mu \mathrm{g} / \mathrm{ml}$. For isolate JMS14, the final concentrations for dimethachlone were $1,10,100,300$, and $600 \mu \mathrm{g} / \mathrm{ml}$, and the final concentrations for prochloraz were $0.0003,0.003,0.03,0.09$, and $0.18 \mu \mathrm{g} / \mathrm{ml}$. Dimethachlone and prochloraz were combined at the following concentrations: 1 (dimethachlone $)+0.0003$ (prochloraz), $10+0.003,100+0.03,300+0.09$, and $600+0.18 \mu \mathrm{g} / \mathrm{ml}$. 
Leaves of about 9 to $12 \mathrm{~cm}$ in diameter were detached from 4 -week-old rapeseed plants growing in the greenhouse at $25^{\circ} \mathrm{C}$. The detached leaves were rinsed with tap water, air dried for $1 \mathrm{~h}$, sprayed with the diluted fungicide solutions using a handheld sprayer $(800 \mathrm{ml}$ in volume, Xinmeir Co., China), air dried for another $1 \mathrm{~h}$, and transferred to $15-\mathrm{cm}$-diameter Petri dishes lined with wetted filter paper to maintain high humidity. Leaves sprayed with $0.1 \%$ Triton X-100 in water were used as the nontreated control. Leaves were then inoculated on the adaxial surface with fresh mycelial plugs cut from the margin of actively growing colonies on PDA without fungicide. The inoculated leaves were incubated at $25^{\circ} \mathrm{C}$ for $48 \mathrm{~h}$ in a growth chamber with relative humidity maintained at approximately $85 \%$. The diameter of each lesion was measured twice at right angles. The experiment was conducted in triplicate (three leaves for each treatment) and repeated independently four times.

Stimulatory effects of spraying dimethachlone and prochloraz mixed at a concentration ratio of $1: 1$ on the virulence of $S$. sclerotiorum to detached rapeseed leaves. The method for this experiment was similar to that described in the previous section. The stock solutions of dimethachlone and prochloraz were first diluted with methanol to the concentrations of $50,100,200$, and $500 \mu \mathrm{g} / \mathrm{ml}$, and these dilutions were further diluted 10 -fold with $0.1 \%$ Triton $\mathrm{X}-100$ in water. The fungicide mixtures contained dimethachlone and prochloraz at $5+5,10+10,20+20$, and $50+50 \mu \mathrm{g} / \mathrm{ml}$. Rapeseed leaves were rinsed, sprayed with fungicide mixtures, inoculated with mycelial plugs, and incubated, and lesion diameters were measured as described above. The experiment was conducted in triplicate (three leaves for each treatment) and repeated independently four times.

Effects of dimethachlone and prochloraz on tolerance of S. sclerotiorum to hydrogen peroxide $\left(\mathrm{H}_{2} \mathrm{O}_{2}\right)$. The method was adopted from Cong et al. (2018) and Di et al. (2015). Fungicide-amended PDA was prepared as described above. For isolate HLJ4, the final concentration of dimethachlone in PDA was $200 \mu \mathrm{g} / \mathrm{ml}$, and the final concentration of prochloraz was $0.04 \mu \mathrm{g} / \mathrm{ml}$. The fungicide mixture in PDA contained dimethachlone at $200 \mu \mathrm{g} / \mathrm{ml}$ and prochloraz at $0.04 \mu \mathrm{g} / \mathrm{ml}$. For isolate JMS14, the final concentrations of dimethachlone and prochloraz in PDA were 100 and $0.03 \mu \mathrm{g} / \mathrm{ml}$, respectively, and the fungicide mixture consisted of dimethachlone at $100 \mu \mathrm{g} / \mathrm{ml}$ and prochloraz at $0.03 \mu \mathrm{g} / \mathrm{ml}$. Mycelia of the two isolates were grown on fungicide-amended PDA at $23^{\circ} \mathrm{C}$ for $48 \mathrm{~h}$, and mycelial plugs were cut from actively growing margins of fungal culture colonies. Mycelial plugs cut from culture colonies grown on PDA amended with the same volume of methanol were used as the nontreated control. The fresh mycelial plugs were inoculated on PDA medium amended with $\mathrm{H}_{2} \mathrm{O}_{2}$ at 0,50 , and $200 \mu \mathrm{g} / \mathrm{ml}$. After the PDA plates had been incubated at $23^{\circ} \mathrm{C}$ for $48 \mathrm{~h}$, the diameter of each mycelial colony was measured twice at right angles. The experiment was conducted in triplicate (three PDA plates for each treatment) and repeated independently three times.

Effects of dimethachlone and prochloraz on mycelial cell membrane permeability. Mycelial cell membrane permeability was measured according to the methods of Lu et al. (2015) and Zhang et al. (2018). PDA medium for isolate HLJ4 was amended with dimethachlone at $50 \mu \mathrm{g} / \mathrm{ml}$, prochloraz at $0.01 \mu \mathrm{g} / \mathrm{ml}$, and concurrently with dimethachlone and prochloraz at 50 and $0.01 \mu \mathrm{g} / \mathrm{ml}$, respectively. For isolate JMS14, the concentrations of dimethachlone and prochloraz in PDA were 30 and $0.01 \mu \mathrm{g} / \mathrm{ml}$, respectively, and the mixture contained dimethachlone at $30 \mu \mathrm{g} / \mathrm{ml}$ and prochloraz at $0.01 \mu \mathrm{g} / \mathrm{ml}$. The fungicide-amended PDA medium at about $55^{\circ} \mathrm{C}$ was poured into 9-cm-diameter Petri dishes, and after being air cooled to room temperature, PDA medium was overlaid with a piece of sterilized cellophane. Mycelial plugs of the two isolates of S. sclerotiorum were inoculated on cellophane-overlaid PDA and incubated at $23^{\circ} \mathrm{C}$ for $48 \mathrm{~h}$. Mycelia grown on PDA amended with methanol at $0.2 \%$ ( $\mathrm{vol} / \mathrm{vol}$ ) were used as the nontreated control. Mycelia actively growing on the outermost approximately $2 \mathrm{~cm}$ in width belt of colonies were harvested by gentle scraping with a sterilized weighing spoon. Approximately $0.20 \mathrm{~g}$ of mycelia per sample was suspended in $35 \mathrm{ml}$ of double-distilled water in a 50 - $\mathrm{ml}$ sterilized beaker, and the beaker was gently shaken for $3 \mathrm{~s}$ by hand. After 5, 10, 20, 40, 60, 80, $100,120,140,160$, and 180 min of suspension, the electrical conductivity of the double-distilled water was measured at room temperature with an electrical conductivity meter (DDS-12DW, Shuanxu Electronics Co., China). After the last measurement, the mycelial suspensions were boiled for $5 \mathrm{~min}$. After the suspensions cooled down to room temperature, the final conductivity was measured. Relative conductivity was calculated by the following formula: relative conductivity $(\%)=$ conductivity/the final conductivity $\times 100 \%$. The experiment was conducted in triplicate and repeated independently three times.

Data processing and analysis. Percent stimulations of virulence were calculated by the following formula: percent stimulation $(\%)=$ [(lesion diameter of the treated - lesion diameter of the control)/ lesion diameter of the control] $\times 100 \%$. Curve modeling was performed according to the method of Deng et al. (2001) with the second-order polynomial (quadratic) procedure using Microsoft Excel software (version 2010, Microsoft, U.S.A.). Following analysis of variance, the Dunnett multiple-comparison procedure in SPSS software (Statistical Product and Service Solutions, version 21.0, SPSS, U.S.A.) with the nontreated control as the control group was used to test significant differences in colony diameters on PDA amended with $\mathrm{H}_{2} \mathrm{O}_{2}$.

\section{Results}

Stimulatory effects of dimethachlone and prochloraz on mycelial growth of $\boldsymbol{S}$. sclerotiorum on PDA. Prochloraz alone in PDA showed similar stimulation amplitude for mycelial growth of the two isolates tested. The maximum percent stimulations were 11.1 and $9.3 \%$ for isolates HLJ4 and JMS14, respectively (Fig. 1). However, there was a huge difference in stimulation amplitude for dimethachlone alone between the two isolates. The maximum percent stimulations for isolates HLJ4 and JMS14 were 19.3 and $80.6 \%$, respectively. For mixtures of prochloraz and dimethachlone, the difference in stimulation amplitude was obvious between the two isolates. For isolate HLJ4, the maximum percent stimulation was only $9.3 \%$. For isolate JMS14, the maximum growth stimulation for the mixture was $48.1 \%$, occurring with dimethachlone at $10 \mu \mathrm{g} / \mathrm{ml}$ and prochloraz at $0.003 \mu \mathrm{g} / \mathrm{ml}$. Dimethachlone alone at $10 \mu \mathrm{g} / \mathrm{ml}$ had the $\max -$ imum stimulation of $80.6 \%$, and prochloraz alone at $0.003 \mu \mathrm{g} / \mathrm{ml}$ had no obvious effect on mycelial growth of isolate JMS14. These results indicate that the interactions between dimethachlone and prochloraz were not effect-additive with respect to mycelial growth stimulation.

Stimulatory effects of the first-generation growth on fungicideamended PDA on the second-generation mycelial growth on PDA. After the stimulated and inhibited mycelia growing on PDA amended with fungicide (as the first generation) were subcultured on PDA without fungicide (as the second generation), significant mycelial growth stimulations were found (Fig. 2). For isolate HLJ4, mycelia with the highest stimulation for the first-generation growth on PDA amended with dimethachlone at $50 \mu \mathrm{g} / \mathrm{ml}$ showed relatively low stimulation for the second-generation growth. At relatively high concentrations, dimethachlone at 200 and $400 \mu \mathrm{g} / \mathrm{ml}$ and prochloraz at 0.04 and $0.08 \mu \mathrm{g} / \mathrm{ml}$ alone, and their mixtures inhibited the first-generation mycelial growth but increased the secondgeneration mycelial growth, and the stimulation amplitudes were even greater than those of low concentrations. For isolate JMS14, the stimulation trend for the second-generation mycelial growth was similar to that of isolate HLJ4, but with higher stimulation amplitudes. The mixture of dimethachlone at $100 \mu \mathrm{g} / \mathrm{ml}$ and prochloraz at $0.03 \mu \mathrm{g} / \mathrm{ml}$ inhibited the first-generation mycelial growth by $59.4 \%$ (Fig. 1) but increased the second-generation mycelial growth by $73.0 \%$ (Fig. 2).

Stimulatory effects of mycelial growth on fungicide-amended PDA on the virulence to rapeseed leaves. Growing on fungicideamended PDA had significant stimulatory effects on the virulence of S. sclerotiorum to rapeseed leaves (Fig. 3). For isolate HLJ4, the maximum virulence stimulation amplitudes for the two fungicides were higher than those of mycelial growth on PDA. The maximum 
virulence stimulation amplitude for the mixture of dimethachlone and prochloraz was slightly higher than those of dimethachlone and prochloraz alone. For isolate JMS14, the maximum virulence stimulation amplitudes for prochloraz alone and for the mixture of dimethachlone and prochloraz were greater than their respective maximum stimulation amplitude for the first-generation mycelial growth on PDA. After the growth-inhibited mycelia on PDA amended with fungicide were inoculated on rapeseed leaves, significant virulence stimulations were found compared with the nontreated control.

Stimulatory effects of spraying dimethachlone and prochloraz mixed at their respective stimulatory concentrations on the virulence of $\boldsymbol{S}$. sclerotiorum to rapeseed leaves. Spraying dimethachlone, prochloraz, and their mixture on rapeseed leaves had significant stimulatory effects on the virulence of S. sclerotiorum, and the fitted stimulation curves for dimethachlone and the mixture of dimethachlone and prochloraz were typical, parabolic hormetic curves (Fig. 4). For isolate HLJ4, the mixture of dimethachlone and prochloraz was similar to dimethachlone alone in stimulation amplitude, but for isolate JMS14, the maximum stimulation amplitude for the fungicide mixture was much higher than that of dimethachlone alone. For both of the two isolates, the concentrations of fungicide mixtures eliciting the maximum stimulation shifted to the left compared with dimethachlone alone, indicating doseadditive interactions between dimethachlone and prochloraz with respect to virulence stimulation.

Stimulatory effects of spraying dimethachlone and prochloraz mixed at a concentration ratio of $1: 1$ on the virulence to rapeseed leaves. Spraying dimethachlone at concentrations from 5 to $50 \mu \mathrm{g} / \mathrm{ml}$ showed significant stimulatory effects on the virulence of the two isolates (Fig. 5). However, spraying prochloraz at the same concentration range showed inhibitory effects on disease lesion development, and prochloraz at $50 \mu \mathrm{g} / \mathrm{ml}$ inhibited the virulence of isolate HLJ4 by $64.3 \%$. The mixture of dimethachlone and prochloraz at concentrations of $20+20$ and $50+50 \mu \mathrm{g} / \mathrm{ml}$ showed higher percent inhibitions than those of prochloraz alone, demonstrating that dimethachlone at the virulence-stimulating concentrations enhanced inhibitory effects of prochloraz. For isolate JMS14, dimethachlone alone at $50 \mu \mathrm{g} / \mathrm{ml}$ increased virulence by $72.4 \%$, and prochloraz at
$50 \mu \mathrm{g} / \mathrm{ml}$ inhibited virulence by $86.5 \%$. However, the mixture of dimethachlone and prochloraz both at $50 \mu \mathrm{g} / \mathrm{ml}$ reduced virulence by $98.2 \%$. These results indicated again that the interactions between dimethachlone and prochloraz were dose-additive rather than effect-additive with respect to inhibitory effect on the virulence of S. sclerotiorum.

Effects of dimethachlone and prochloraz on the sensitivity of $S$. sclerotiorum to $\mathbf{H}_{2} \mathbf{O}_{2}$. After mycelia were grown for $48 \mathrm{~h}$ on PDA amended with dimethachlone at $200 \mu \mathrm{g} / \mathrm{ml}$ and prochloraz at $0.04 \mu \mathrm{g} / \mathrm{ml}$ for isolate HLJ4, and with dimethachlone at $100 \mu \mathrm{g} / \mathrm{ml}$ and prochloraz at $0.03 \mu \mathrm{g} / \mathrm{ml}$ for isolate JMS14, tolerance to $\mathrm{H}_{2} \mathrm{O}_{2}$ increased, and the increases were statistically significant (Fig. 6). For isolate HLJ4, the mixture of dimethachlone and prochloraz had a similar increase in $\mathrm{H}_{2} \mathrm{O}_{2}$ tolerance to that of dimethachlone and prochloraz alone. For isolate JMS14, the increase in $\mathrm{H}_{2} \mathrm{O}_{2}$ tolerance for the mixture was significantly lower than that of dimethachlone and prochloraz alone. These results indicate interactions between dimethachlone and prochloraz are not effect-additive with regard to tolerance to $\mathrm{H}_{2} \mathrm{O}_{2}$.

Effects of dimethachlone and prochloraz on mycelial cell membrane permeability. The two isolates displayed similar mycelial cell membrane permeability responses to treatments with dimethachlone and prochloraz (Fig. 7). Dimethachlone (at $50 \mu \mathrm{g} / \mathrm{ml}$ ) increased whereas prochloraz (at $0.1 \mu \mathrm{g} / \mathrm{ml}$ ) decreased cell membrane permeability of the two isolates. Mycelial cell membrane permeability for the treatment with the mixture of dimethachlone and prochloraz lay between those of dimethachlone and prochloraz, indicating an effect-additive interaction between the two fungicides with respect to the effect of fungicides on cell membrane permeability of S. sclerotiorum.

\section{Discussion}

The present study showed that dimethachlone and prochloraz, either alone or in combinations, had significant stimulatory effects on mycelial growth of $S$. sclerotiorum on PDA, but the stimulation amplitudes differed greatly between the two isolates and between the two fungicides. The maximum stimulation amplitude of dimethachlone was much higher than that of prochloraz, and the maximum stimulation amplitude of dimethachlone for isolate JMS14 was
A

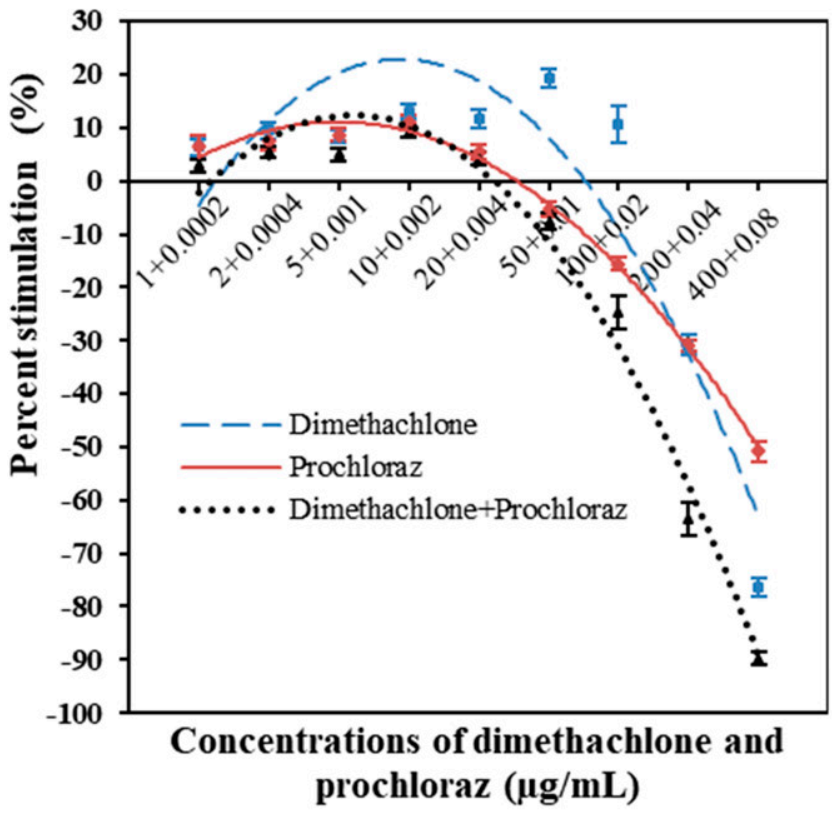

B

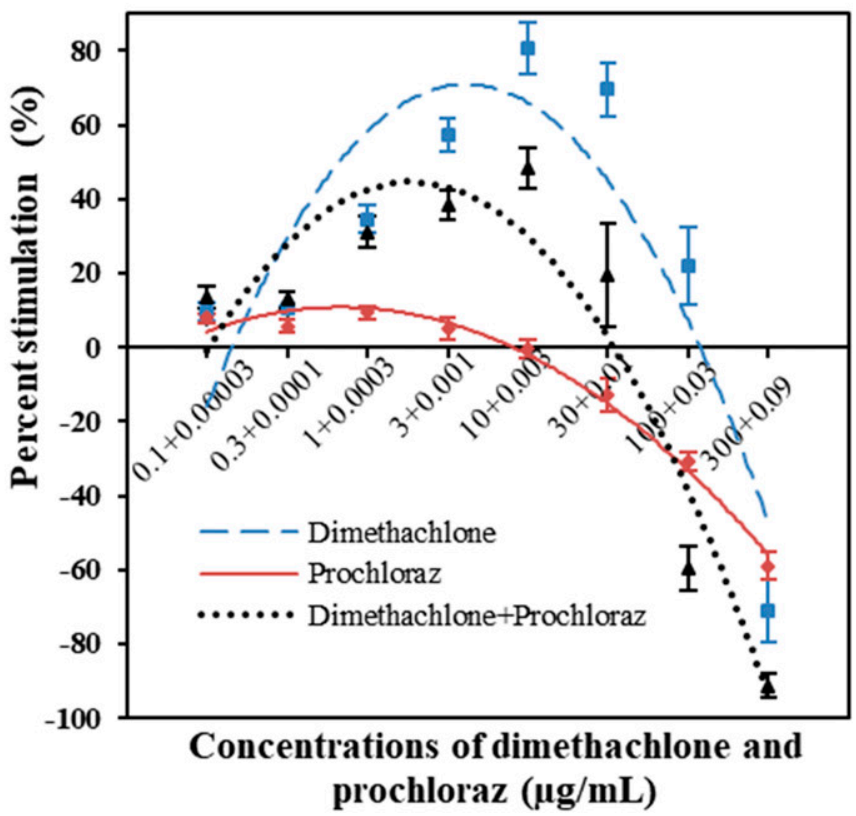

Fig. 1. Stimulatory effects of prochloraz and dimethachlone on mycelial growth of Sclerotinia sclerotiorum isolates HLJ4 (A) and JMS14 (B) on potato dextrose agar. Numbers before and after the plus sign $(+)$ on the $x$ axis denote the concentrations of dimethachlone and prochloraz, respectively. Error bar denotes the standard error of the mean of eight repeated experiments. 
much higher than that of isolate HLJ4. However, for prochloraz, there was no significant difference in maximum stimulation amplitude between the two isolates. In a broad context, the maximum hormetic amplitudes of various endpoints for a large number of structurally diverse chemicals usually lie between approximately 30 and $60 \%$ compared with a nontreated control (Calabrese and
Blain 2011). Our previous study also demonstrated that dimethachlone in PDA increased mycelial growth of S. sclerotiorum isolates LA50R and HF84R and that the maximum stimulation amplitudes were less than $60 \%$ (Zhou et al. 2014a). In the present study, the maximum stimulation amplitude for isolate JMS14 was as high as $80.6 \%$. This higher stimulation amplitude might be
A

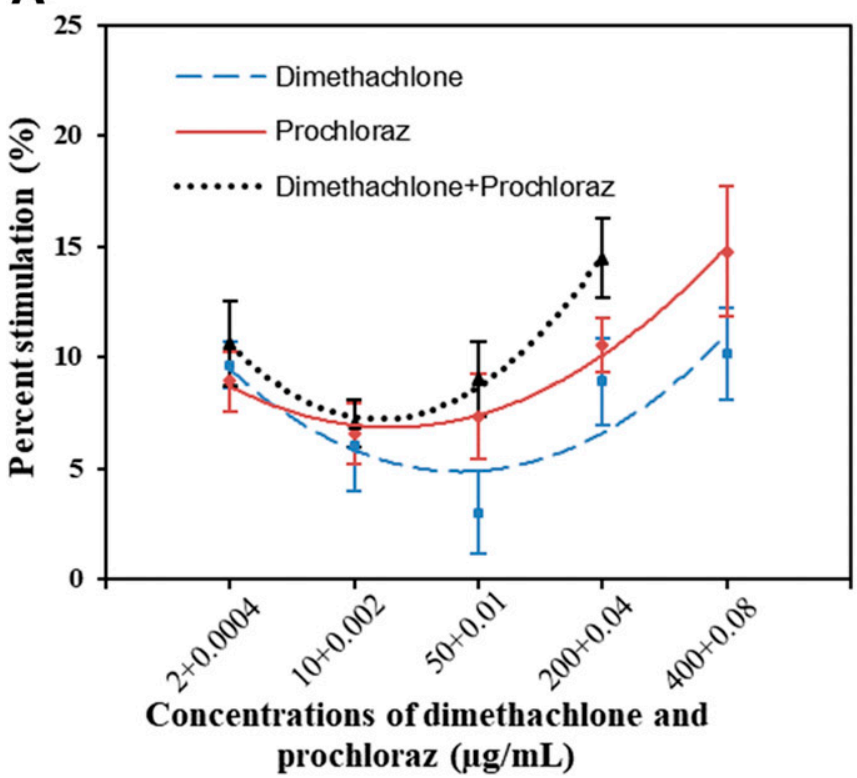

B

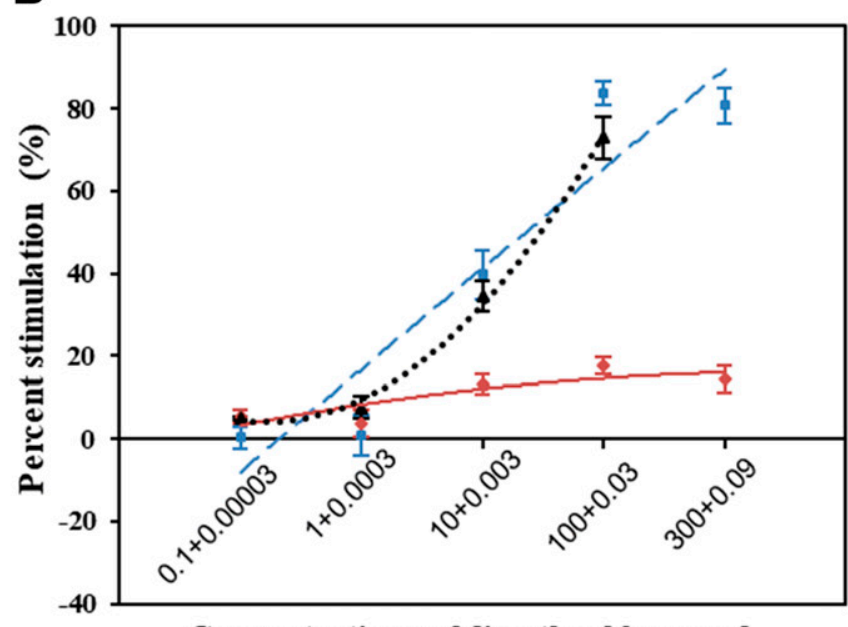

Concentrations of dimethachlone and prochloraz $(\mu \mathrm{g} / \mathrm{mL})$

Fig. 2. Stimulatory effects of growing on potato dextrose agar (PDA) amended with prochloraz and dimethachlone as a pretreatment on second-generation mycelial growth of Sclerotinia sclerotiorum isolates HLJ4 (A) and JMS14 (B) on PDA. Numbers before and after the plus sign (+) on the $x$ axis denote the concentrations of dimethachlone and prochloraz, respectively. Error bar denotes the standard error of the mean of five repeated experiments.

A

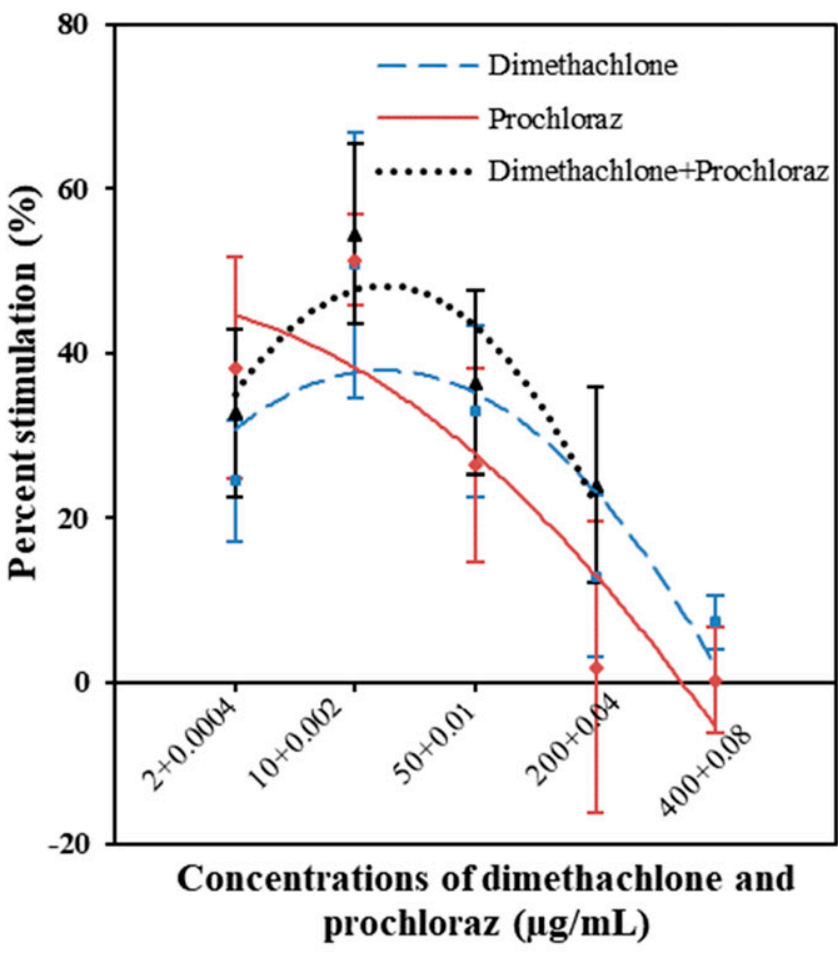

B

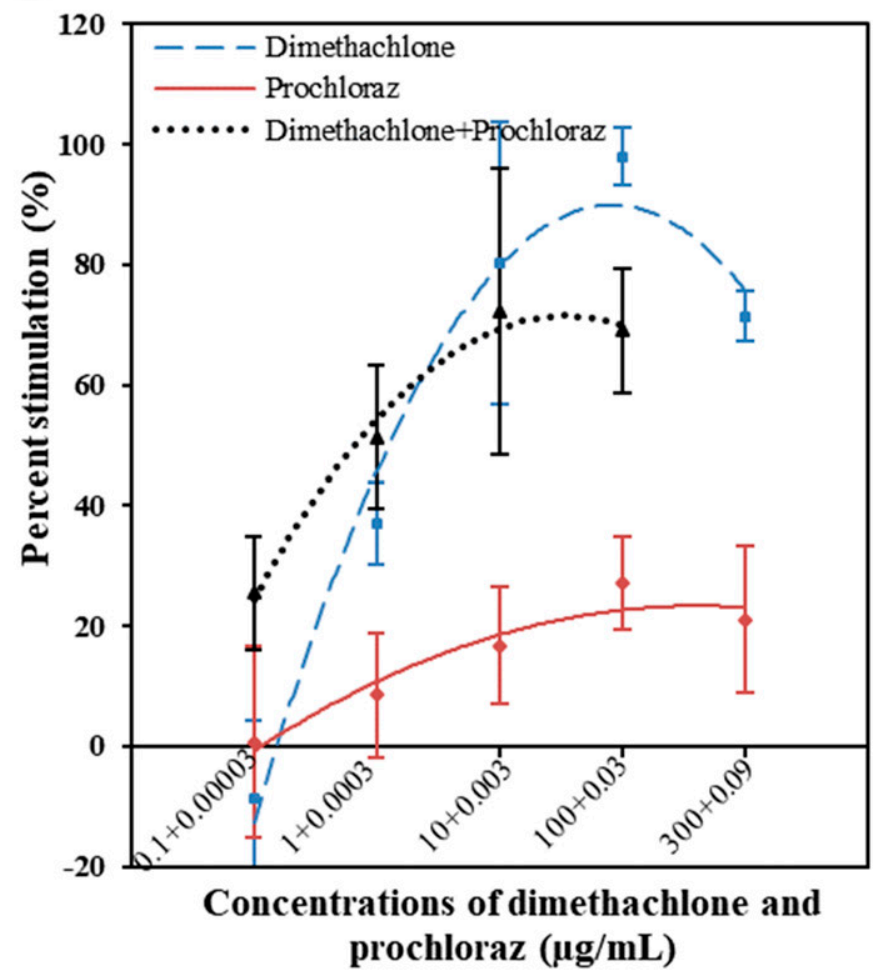

Fig. 3. Stimulatory effects of growing on potato dextrose agar amended with prochloraz and dimethachlone as a pretreatment on the virulence of Sclerotinia sclerotiorum isolates HLJ4 (A) and JMS14 (B) on rapeseed leaves. Numbers before and after the plus sign (+) on the $x$ axis denote the concentrations of dimethachlone and prochloraz, respectively. Error bar denotes the standard error of the mean of five repeated experiments. 
attributed to the relatively slow growth rate of isolate JMS14. The dimethachlone-resistant isolate JMS14 grows much slower than sensitive isolates and also slower than isolate HLJ4 on PDA without any fungicide (data not shown), and dimethachlone may stimulate its growth to potential limitations that are constrained by biological plasticity (Calabrese and Mattson 2011, 2017). When mixed together, dimethachlone did not increase the stimulatory effect of prochloraz on mycelial growth of isolate HLJ4, whereas it increased significantly the stimulatory effect of prochloraz on isolate JMS14.
After the growth-increased and growth-inhibited mycelia were inoculated on PDA and on rapeseed leaves without any fungicide, stimulation on mycelial growth and virulence was still remarkable. In fact, the percent stimulation for the second-generation mycelial growth on PDA was even higher than that for the first-generation mycelial growth on PDA amended with fungicide. The stimulation amplitude for virulence to rapeseed leaves increased substantially compared with the first-generation mycelial growth on PDA amended with fungicide. This phenomenon has been reported in our previous studies on stimulatory effects of dimethachlone (Zhou
A

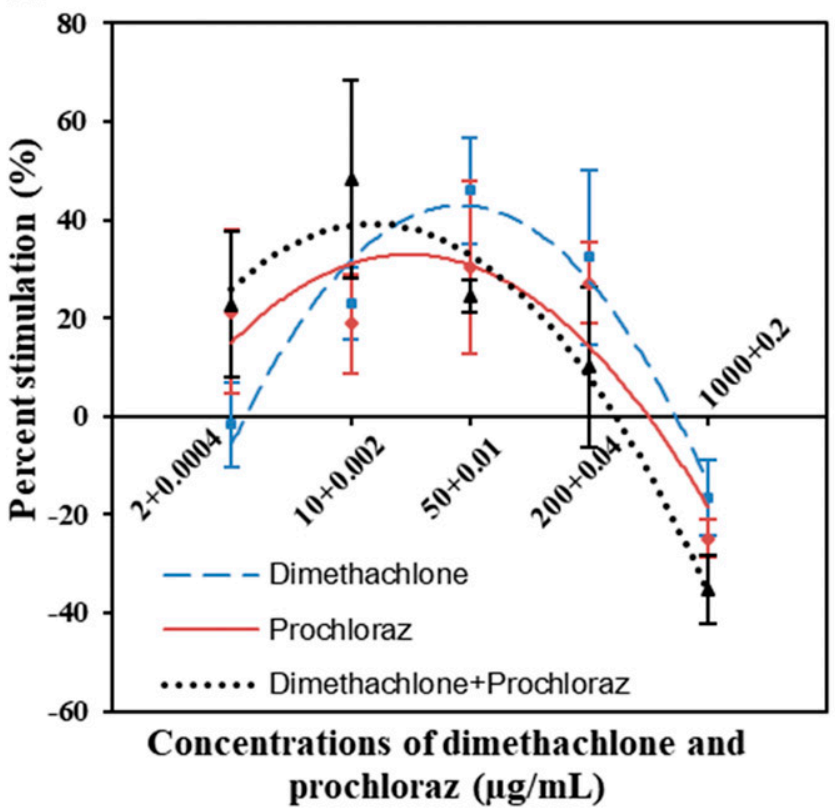

B

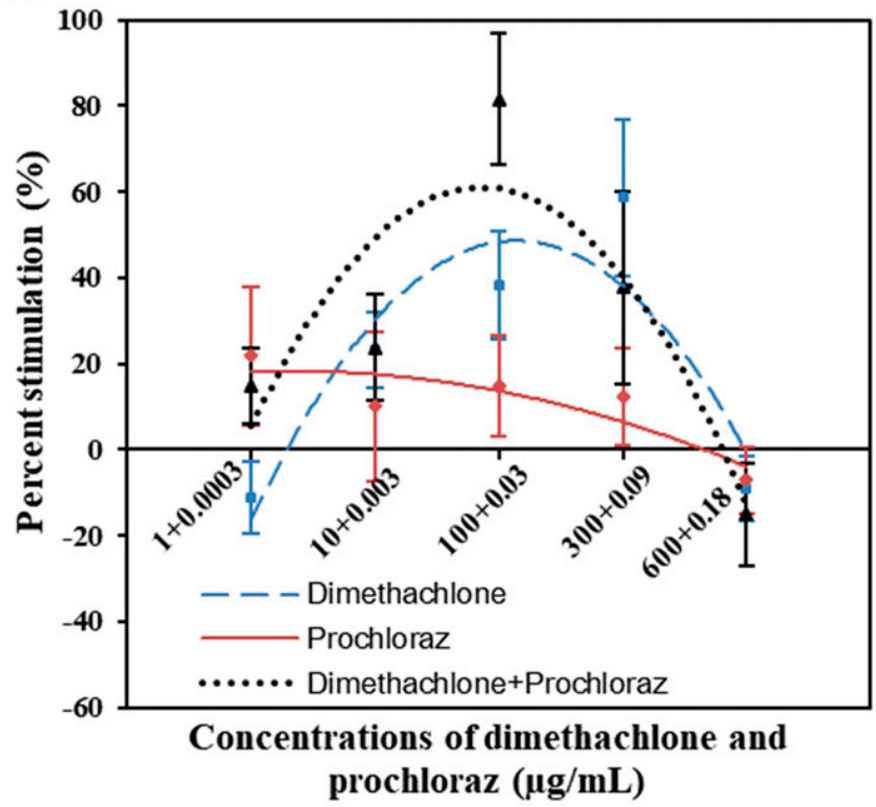

Fig. 4. Stimulatory effects of spraying prochloraz, dimethachlone, and their mixtures on the virulence of Sclerotinia sclerotiorum isolates HLJ4 (A) and JMS14 (B) on rapeseed leaves. Numbers before and after the plus sign $(+)$ on the $x$ axis denote the concentrations of dimethachlone and prochloraz, respectively. Error bar denotes the standard error of the mean of four repeated experiments.

A

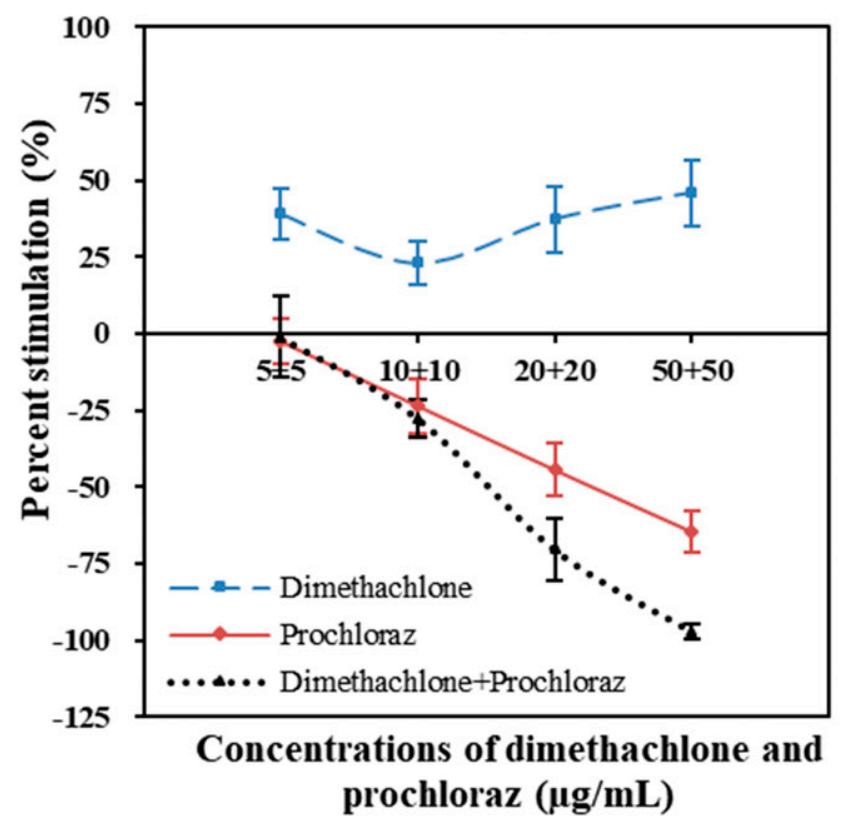

B

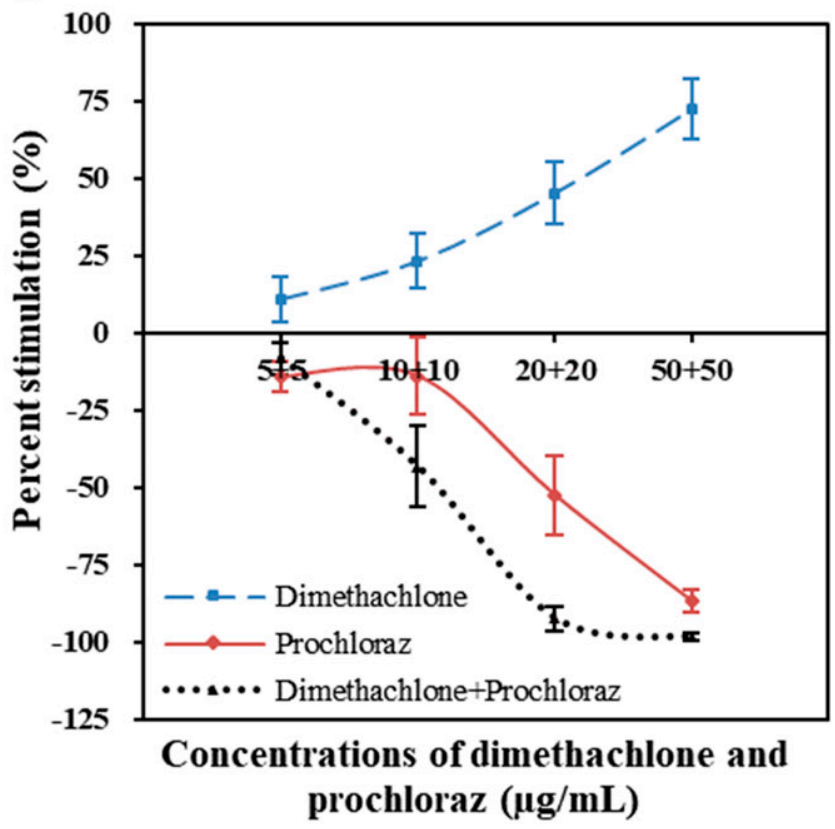

Fig. 5. Stimulatory effects of spraying prochloraz, dimethachlone, and their mixture at the weight ratio of 1:1 on the virulence of Sclerotinia sclerotiorum isolates HLJ4 (A) and JMS14 (B) on rapeseed leaves. Error bar denotes the standard error of the mean of four repeated experiments. 
et al. 2014a) and flusilazole (Lu et al. 2018b) on S. sclerotiorum; however, studies on hormetic effects of carbendazim on $B$. cinerea did not show a similar phenomenon (Cong et al. 2018). Of more concern was the fact that mycelia inhibited by more than 50\% on PDA still showed considerable virulence stimulations. This phenomenon has profound implications for understanding fungicide hormesis and for applications of fungicides in the field. The biological concept of hormesis implies high-dose inhibition and low-dose stimulation. In the present study, dimethachlone alone at 400 and $300 \mu \mathrm{g} / \mathrm{ml}$ for isolates HLJ4 and JMS14, respectively, and prochloraz alone at 0.08 and $0.09 \mu \mathrm{g} / \mathrm{ml}$ for isolates HLJ4 and JMS14, respectively, inhibited mycelial growth by more than $50 \%$. These were highdose inhibitions. However, if subsequent virulence was determined, there were considerable stimulations at these relatively high doses. This implies that high doses of chemicals might have significant inhibitory effects on one endpoint, but for another endpoint, if tested subsequently, the high-dose chemical might still have significant hormetic effects. In the field, fungicides are applied at relatively high doses to control plant pathogens, and the pathogens may be killed or inhibited, but the inhibited pathogen may become much more virulent to the host plant when it infects new healthy plants in the absence of fungicide. This also implies that our common, successful fungicide application practices for field control of plant pathogens may hide considerable hormesis. Unfortunately, the hidden hormetic effects are adverse to the control of plant pathogens and are liable to be ignored by farmers.

Spraying dimethachlone, prochloraz, and their mixtures showed obvious stimulatory effects on the virulence of S. sclerotiorum. The stimulatory dose range of dimethachlone for the two isolates was around 2 to $200 \mu \mathrm{g} / \mathrm{ml}$, and the stimulatory dose range of
A

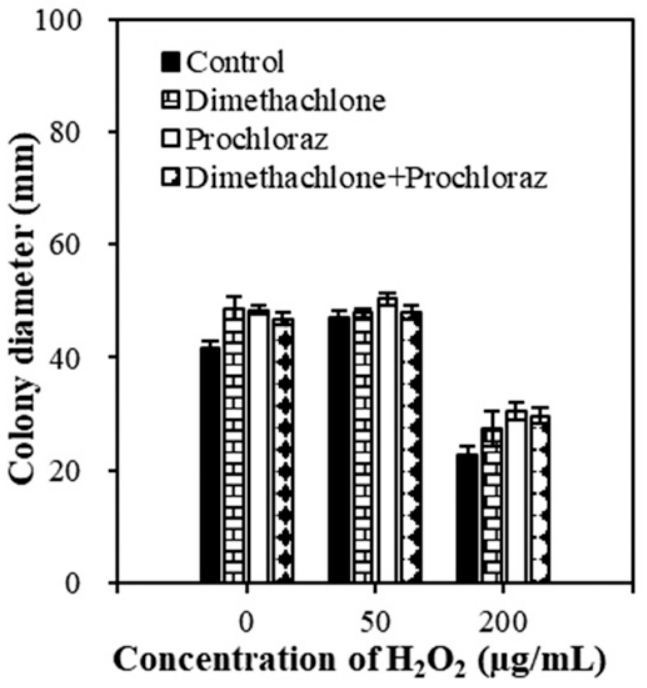

B

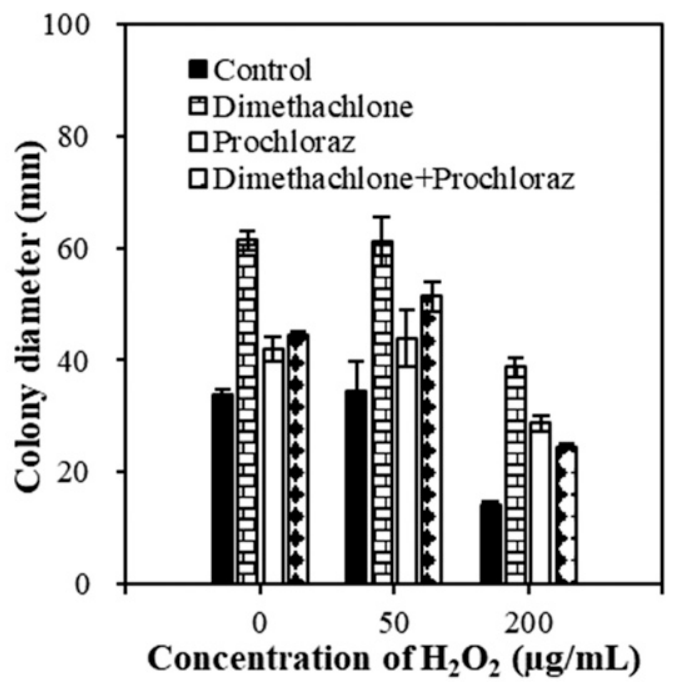

Fig. 6. Effects of growing on potato dextrose agar (PDA) supplemented with prochloraz, dimethachlone, and their mixtures on the sensitivity of Sclerotinia sclerotiorum isolates $\mathrm{HLJ4}(\mathbf{A})$ and JMS14 (B) to $\mathrm{H}_{2} \mathrm{O}_{2}$. For isolate $\mathrm{HLJ4}$, the concentrations of dimethachlone and prochloraz in PDA were 200 and $0.04 \mu \mathrm{g} / \mathrm{ml}$, respectively. For isolate JMS14, the concentrations of dimethachlone and prochloraz were 100 and $0.03 \mu \mathrm{g} / \mathrm{ml}$, respectively. Error bar denotes the standard error of the mean of three repeated experiments.

A

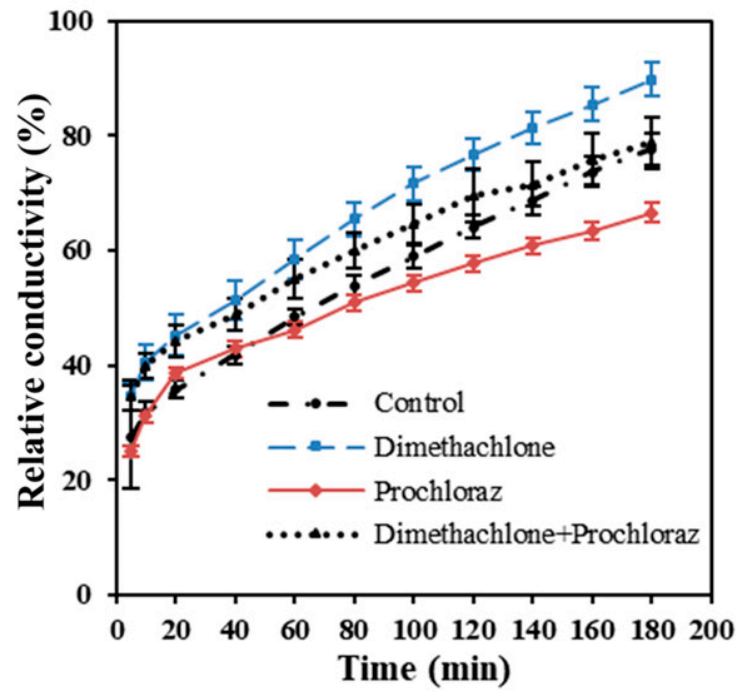

B

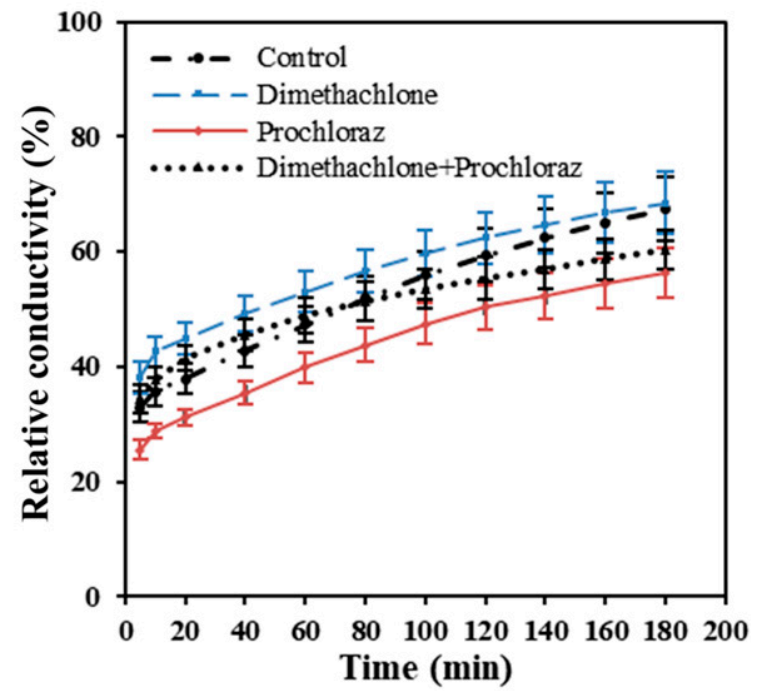

Fig. 7. Effects of prochloraz, dimethachlone, and their mixtures on cell membrane permeability of Sclerotinia sclerotiorum isolates HLJ4 (A) and JMS14 (B). For isolate HLJ4, the concentrations of dimethachlone and prochloraz in potato dextrose agar were 50 and $0.01 \mu \mathrm{g} / \mathrm{ml}$, respectively. For isolate JMS14, the concentrations of dimethachlone and prochloraz were 30 and $0.01 \mu \mathrm{g} / \mathrm{ml}$, respectively. Error bar denotes the standard error of the mean of three repeated experiments. 
prochloraz was about 0.0003 to $0.09 \mu \mathrm{g} / \mathrm{ml}$. The fitted models for stimulatory effects of prochloraz were not typical, parabolic curves, which could be owing to low concentrations of prochloraz and experimental errors. Dimethachlone and prochloraz were mixed at their respective virulence-stimulating concentrations, and the mixture showed typical hormetic curves. The maximum stimulation amplitude of the mixture of dimethachlone and prochloraz was not significantly higher than that of dimethachlone alone, but the stimulatory dose zone for the mixture of fungicides shifted to the left, indicating dose-additive interactions between dimethachlone and prochloraz with respect to stimulatory effects. Chemical interactions have been well established in toxicology, but few studies on chemical interactions in the context of hormesis have been reported (Calabrese and Mattson 2017). Carbendazim and iprodione also showed doseadditive interactions with regard to stimulatory effects on the virulence of B. cinerea (data not shown). The present study also investigated the stimulatory effects of the mixture of dimethachlone and prochloraz mixed at the concentration ratio of $1: 1$. Dimethachlone at 5 to $50 \mu \mathrm{g} / \mathrm{ml}$ showed significant stimulatory effects on the virulence of $S$. sclerotiorum, whereas prochloraz at the same dose range had inhibitory effects on the virulence. For the mixture of dimethachlone and prochloraz mixed at a concentration ratio of 1:1, significant inhibitory effects were found, and the percent inhibitions at relatively high concentrations were higher than that of prochloraz. These results indicate that the interaction between dimethachlone and prochloraz was dose-additive rather than effect (amplitude)additive with respect to inhibitory effects on the virulence of $S$. sclerotiorum. It should be noted that dimethachlone and prochloraz at the same dose range of 5 to $50 \mu \mathrm{g} / \mathrm{ml}$ showed completely opposite effects on the virulence of $S$. sclerotiorum, and the stimulatory dose zone for dimethachlone was about 10,000-fold higher than that of prochloraz. The reason was that the two isolates used in the present study were resistant to dimethachlone but sensitive to prochloraz.

Stimulations on mycelial growth and virulence are external manifestations of fungicide hormesis. Elucidating the underlying mechanisms will deepen our comprehensive understandings of hormetic effects of fungicides. Increased tolerance of fungal pathogens to $\mathrm{H}_{2} \mathrm{O}_{2}$ may facilitate their successful infections, because $\mathrm{H}_{2} \mathrm{O}_{2}$ may represent reactive oxygen species released by host plants to combat early infections of pathogens (Williams et al. 2011; Wojtaszek 1997). Our earlier studies showed that dimethachlone increases tolerance of $S$. sclerotiorum to $\mathrm{H}_{2} \mathrm{O}_{2}$, and this may be one of the mechanisms for the hormetic effects of dimethachlone on the virulence of this pathogen (Zhou et al. 2014a). However, our subsequent studies showed that carbendazim at the virulence-stimulating doses does not promote tolerance of $S$. sclerotiorum (Di et al. 2015) and B. cinerea (Cong et al. 2018) to $\mathrm{H}_{2} \mathrm{O}_{2}$. The present study showed that dimethachlone, prochloraz, and their mixture increased tolerance to $\mathrm{H}_{2} \mathrm{O}_{2}$, and this might partially explain the hormetic effects of these fungicides on the virulence of $S$. sclerotiorum. Although cell membrane permeability has no direct correlation with the virulence of fungal pathogens, studies on the effects of dimethachlone and prochloraz on cell membrane permeability may provide us with clues of interactions of fungicide mixtures with respect to influence on the physiology of fungal pathogens. The results showed clearly that dimethachlone and prochloraz had effect-additive interactions with respect to effects on cell membrane permeability. These results were completely contrary to the dose-additive interactions with regard to effects on the virulence. This indicates that chemical interactions in hormesis are strikingly different from that of traditional toxicology. As studied with the memory-enhancing drugs (Calabrese 2008; Calabrese and Mattson 2017), additive and even synergic interactions could occur at low stimulation zones. However, as the stimulatory effects approach the maximum amplitude, the additive or synergistic interactions become progressively less effective (Calabrese and Mattson 2017).

\section{Literature Cited}

Audenaert, K., Callewaert, E., Höfte, M., Saeger, S. D., and Haesaert, G. 2010.

Hydrogen peroxide induced by the fungicide prothioconazole triggers deoxynivalenol (DON) production by Fusarium graminearum. BMC Microbiol. 10:112.

Baraldi, E., Mari, M., Chierici, E., Pondrelli, M., Bertolini, P., and Pratella, G. C. 2003. Studies on thiabendazole resistance of Penicillium expansum of pears: Pathogenic fitness and genetic characterization. Plant Pathol. 52:362-370.

Boland, G. J., and Hall, R. 1994. Index of plant hosts of Sclerotinia sclerotiorum. Can. J. Plant Pathol. 16:93-108.

Bolton, D. M., Thomma, B. P., and Nelson, B. D. 2006. Sclerotinia sclerotiorum (Lib.) de Bary: Biology and molecular traits of a cosmopolitan pathogen. Mol. Plant Pathol. 7:1-16.

Brent, K. J., and Hollomon, D. W. 2007. Fungicide Resistance in Crop Pathogens: How Can It Be Managed? FRAC Monograph No. 1, 2nd Ed. Brussels, Belgium. http://www.frac.info/docs/default-source/publications/monographs/ monograph-1.pdf?sfvrsn=769d419a 8

Calabrese, E. J. 2008. Hormesis and mixtures. Toxicol. Appl. Pharmacol. 229: 262-263.

Calabrese, E. J. 2013. Hormetic mechanisms. Crit. Rev. Toxicol. 43:580-606.

Calabrese, E. J. 2015a. Hormesis: Principles and applications. Homeopathy 104: 69-82.

Calabrese, E. J. 2015b. Hormesis within a mechanistic context. Homeopathy 104: 90-96.

Calabrese, E. J. 2016a. Preconditioning is hormesis part I: Documentation, dose-response features and mechanistic foundations. Pharmacol. Res. 110:242-264.

Calabrese, E. J. 2016b. Preconditioning is hormesis part II: How the conditioning dose mediates protection: Dose optimization within temporal and mechanistic frameworks. Pharmacol. Res. 110:265-275.

Calabrese, E. J., and Blain, R. B. 2011. The hormesis database: The occurrence of hormetic dose responses in the toxicological literature. Regul. Toxicol. Pharmacol. 61:73-81.

Calabrese, E. J., and Mattson, M. P. 2011. Hormesis provides a generalized quantitative estimate of biological plasticity. J. Cell Commun. Signal. 5:25-38

Calabrese, E. J., and Mattson, M. P. 2017. How does hormesis impact biology, toxicology, and medicine? NPJ Aging Mech. Dis. 3:13.

Cong, M. L., He, S., Ma, H. J., Li, G. Q., and Zhu, F. X. 2018. Hormetic effects of carbendazim on the virulence of Botrytis cinerea. Plant Dis. 102:886-891.

Deng, C., Graham, R., and Shukla, R. 2001. Detecting and estimating hormesis using a model-based approach. Hum. Ecol. Risk Assess. 7:849-866.

Derbyshire, M. C., and Denton-Giles, M. 2016. The control of Sclerotinia stem rot on oilseed rape (Brassica napus): Current practices and future opportunities. Plant Pathol. 65:859-877.

Di, Y. L., Cong, M. L., Zhang, R., and Zhu, F. X. 2016a. Hormetic effects of trifloxystrobin on aggressiveness of Sclerotinia sclerotiorum. Plant Dis. 100: 2113-2118.

Di, Y. L., Lu, X. M., Zhu, Z. Q., and Zhu, F. X. 2016b. Time-course of carbendazim stimulation on pathogenicity of Sclerotinia sclerotiorum indicates a direct stimulation mechanism. Plant Dis. 100:1454-1459.

Di, Y. L., Zhu, Z. Q., Lu, X. M., and Zhu, F. X. 2015. Pathogenicity stimulation of Sclerotinia sclerotiorum by subtoxic doses of carbendazim. Plant Dis. 99: $1342-1346$

Flores, F. J., and Garzón, C. D. 2013. Detection and assessment of chemical hormesis on the radial growth in vitro of oomycetes and fungal plant pathogens. Dose Response 11:361-373.

Garzón, C. D., and Flores, F. J. 2013. Hormesis: Biphasic dose-responses to fungicides in plant pathogens and their potential threat to agriculture. Pages 311-328 in: Fungicides: Showcases of Integrated Plant Disease Management from Around The world. M. Nita, ed. INTECH, London, U.K.

Garzón, C. D., Molineros, J. E., Yanez, J. M., Flores, F. J., Jimenez-Gasco, M. M., and Moorman, G. W. 2011. Sublethal doses of mefenoxam enhance Pythium damping-off of geranium. Plant Dis. 95:1233-1238.

Lu, X. M., He, S., Ma, H. J., Li, J. H., and Zhu, F. X. 2018a. Hormetic effects of flusilazole preconditioning on mycelial growth and virulence of Sclerotinia sclerotiorum. Plant Dis. 102:1165-1170.

Lu, X. M., Zhang, R., Cong, M. L., Li, J. H., and Zhu, F. X. 2018b. Stimulatory effects of flusilazole on virulence of Sclerotinia sclerotiorum. Plant Dis. 102: 197-201.

Lu, X. M., Zhu, Z. Q., Di, Y. L., and Zhu, F. X. 2015. Baseline sensitivity and toxic action of flusilazole to Sclerotinia sclerotiorum. Crop Prot. 78:92-98.

Ma, H. X., Feng, X. J., Chen, Y., Chen, C. J., and Zhou, M. G. 2009. Occurrence and characterization of dimethachlon insensitivity in Sclerotinia sclerotiorum in Jiangsu Province of China. Plant Dis. 93:36-42.

Pan, Y. L. 1998. The resistance of Sclerotinia sclerotiorum of rape to carbendazim and its management. Jiangsu J. Agric. Sci. 14:159-163.

Ren, L., Chen, K. R., Wang, C. Y., Luo, L. X., Jia, J. G., Wang, J., and Fang, X. P. 2010. Potential of prochloraz-manganese chloride in controlling Sclerotinia stem rot of oilseed rape. Sci. Agric. Sin. 43:4183-4191

Shi, Z. Q., Zhou, M. G., and Ye, Z. Y. 2000. Resistance of Sclerotinia sclerotiorum to carbendazim and dimethachlon. Chin. J. Oil Crop Sci. 22:54-57.

Sun, Z. X., Deng, L., Zhang, C. Q., and Zhou, Y. 2015. Toxicity of several fungicides and chemical mixture against Sclerotinia sclerotiorum. Hubei Agric. Sci. 54:1606-1608

Vinggaard, A. M., Hass, U., Dalgaard, M., Andersen, H. R., Bonefeld-Jørgensen, E., Christiansen, S., Laier, P., and Poulsen, M. E. 2006. Prochloraz: An 
imidazole fungicide with multiple mechanisms of action. Int. J. Androl. 29: 186-192.

Williams, B., Kabbage, M., Kim, H. J., Britt, R., and Dickman, M. B. 2011. Tipping the balance: Sclerotinia sclerotiorum secreted oxalic acid suppresses host defenses by manipulating the host redox environment. PLoS Pathog 7: e1002107.

Wojtaszek, P. 1997. Oxidative burst: An early plant response to pathogen infection. Biochem. J. 322:681-692.

Zhang, R., Xu, Q. R., Zhang, Y. C., and Zhu, F. X. 2018. Baseline sensitivity and toxic actions of prochloraz to Sclerotinia sclerotiorum. Plant Dis. 102: 2149-2157.

Zhang, S., Panaccione, S. G., and Gallegly, M. E. 1997. Metalaxyl stimulation of growth of isolates of Phytophthora infestans. Mycologia 89:289-292.
Zhang, X. L., Sun, X. M., Zhang, G. F., Yi, H. J., and Qiang, Y. 2003. Preliminary report on the monitoring of the resistance of Sclerotinia sclerotiorum to carbendazim and its integrated management. Chin. J. Pestic. Sci. Adm. 24 18-22.

Zhou, F., Liang, H. J., Di, Y. L., You, H., and Zhu, F. X. 2014a. Stimulatory effects of sublethal doses of dimethachlon on Sclerotinia sclerotiorum. Plant Dis. 98: $1364-1370$

Zhou, F., Zhang, X. L., Li, J. L., and Zhu, F. X. 2014b. Dimethachlon resistance in Sclerotinia sclerotiorum in China. Plant Dis. 98:1221-1226.

Zhou, F., Zhu, F. X., Zhang, X. L., and Zhang, A. S. 2014c. First report of dimethachlon resistance in field isolates of Sclerotinia sclerotiorum on oilseed rape in Shaanxi Province of northwestern China. Plant Dis. 98: 568. 